\title{
The Influence of Lattice Defects on the Ground-State Properties of the Falicov-Kimball Model in Two Dimensions
}

\author{
M. ŽondA, P. FARKAŠOVSKÝ AND H. ČENČARIKOVÁ \\ Institute of Experimental Physics, Slovak Academy of Sciences \\ Watsonová 47, 04101 Košice, Slovakia
}

The influence of lattice defects (vacancies) on the ground-state properties of the spinless Falicov-Kimball model is studied by a well-controlled numerical method in two dimensions. It is shown that in the presence of vacancies (distributed randomly) the ground states of the Falicov-Kimball model are phase separated for small $f$-electron concentrations $n_{f}$ and exhibit the long-range order for $n_{f}$ near the half-filled band case $n_{f}=1 / 2$. In addition, the dependence of average $f$-orbital occupancy on the concentration of vacancies is calculated for a wide range of model parameters. The resultant behaviours are used to interpret the experimental data obtained for the mixed-valence system $\mathrm{Sm}_{1-x} \mathrm{~B}_{6}$.

PACS numbers: 75.10.Lp, 71.27.+a, 71.28.+d, 71.30.+h

\section{Introduction}

The Falicov-Kimball model (FKM) [1] was introduced in 1969 to describe metal-insulator transitions in rare-earth and transition-metal compounds and later it has been used successfully to study a great variety of many-body effects, of which valence transitions, charge-density waves and electronic ferroelectricity are the most common examples $[2,3]$. The Hamiltonian of the model can be written as a sum of three terms

$$
H=\sum_{\langle i, j\rangle} t_{i j} d_{i}^{+} d_{j}+U \sum_{i} f_{i}^{+} f_{i} d_{i}^{+} d_{i}+E_{f} \sum_{i} f_{i}^{+} f_{i},
$$

where $f_{i}^{+}, f_{i}$ are the creation and annihilation operators for an electron in the localized state at lattice site $i$ with binding energy $E_{f}$ and $d_{i}^{+}, d_{i}$ are the creation and annihilation operators of the $d$-electrons hopping between the nearest-neighbour sites with hopping probability $t$. The second term represents the on-site Coulomb interaction between the $d$ and $f$ electrons. 
In the present paper we study the influence of lattice defects (vacancies) on the ground state (GS) of the spinless FKM with the aim to describe the valence transition behaviour of $\mathrm{Sm}$ ions in the $\mathrm{Sm}_{1-x} \mathrm{~B}_{6}$ compound. The defect is represented here by a vacancy without any additional impact on the lattice structure. This is a good approximation because the crystal structure of non-stoichiometric compound $\mathrm{Sm}_{1-x} \mathrm{~B}_{6}$ remains stable over a broad range of vacancy concentrations (up to $30 \%$ ) [4, 5].

From the numerical point of view there is only one fundamental difference between the FKM with and without vacancies, and namely, that in the model with vacancies the hopping matrix amplitudes should be changed in such a way that the electron transitions to the vacant sites are forbidden, i.e., $t_{i j}=0$ if $i$ or $j$ represents the position of vacant site. Since in the spinless version of the FKM the operator $f_{i}^{+} f_{i}$ commutes with the total Hamiltonian (1), it can be replaced by a classical variable $w_{i}$, taking only two values: 1 or 0 , according to whether or not the site $i$ is occupied by the localised $f$ electron. Then the Hamiltonian (1) can be written as $H=\sum_{\langle i, j\rangle} h_{i j} d_{i}^{+} d_{j}+E_{f} \sum_{i} w_{i}$, where $h_{i j}(w, v)=t_{i j}+U w_{i} \delta_{i j}$. This Hamiltonian is for a given $f$-electron configuration $w$ and a given distribution of vacancies $v$ the second-quantised version of the single-particle Hamiltonian, so the investigation of the model is reduced to the investigation of the spectrum of $h$ for different $w$ and $v$. We consider only the case $N_{f}+N_{d}+N_{v}=L$ (where $N_{f}, N_{d}$, $N_{v}$ and $L$ are the total number of $f$ electrons, $d$ electrons, vacancies and lattice sites, respectively), which is the point of special interest for the valence transitions, caused by promotion of electrons from the localised $f$ orbitals $\left(f^{n} \rightarrow f^{n-1}\right)$ to the conduction band states.

\section{Results and discussion}

To study the GS properties of the model we have used a well-controlled numerical method elaborated recently by one of the present authors [6]. The numerical calculations have been done for a wide range of the Coulomb interactions in order to represent the typical behaviour of the model in the weak $(U=0.5,1$ and 2), intermediate $(U=4)$ and strong coupling limit $(U=8,10)$. At fixed $N_{\mathrm{v}}$ the GS configurations corresponding to $N_{f}=0,1 \ldots \frac{L-N_{\mathrm{v}}}{2}$ were calculated for a set of hundred random distributions of vacancies for each selected $U$. The same procedure was repeated on several different clusters and it was found that the main features of the GS configurations hold on all examined lattices and thus can be used satisfactorily to represent the behaviour of macroscopic systems.

The representative types of GS configurations are displayed in Fig. 1. We have found that for the weak interactions, small densities of vacancies $n_{\mathrm{v}}=\frac{N_{\mathrm{v}}}{L}$ and small densities of $f$ electrons $n_{f}=\frac{N_{f}}{L-N_{\mathrm{v}}}\left(n_{\mathrm{v}} \leq 1 / 8, n_{f}<1 / 12\right.$ for $U \leq 1$ and $n_{\mathrm{v}} \leq 1 / 8, n_{f}<1 / 16$ for $U=2$ ) the GS configurations of the model are the $n$-molecular phases (the four-molecular phases and the two-molecular phases). In the area of $1 / 12<n_{f}<1 / 2$ for $U \leq 1$ and $1 / 16<n_{f}<1 / 2$ for $U=2$ 


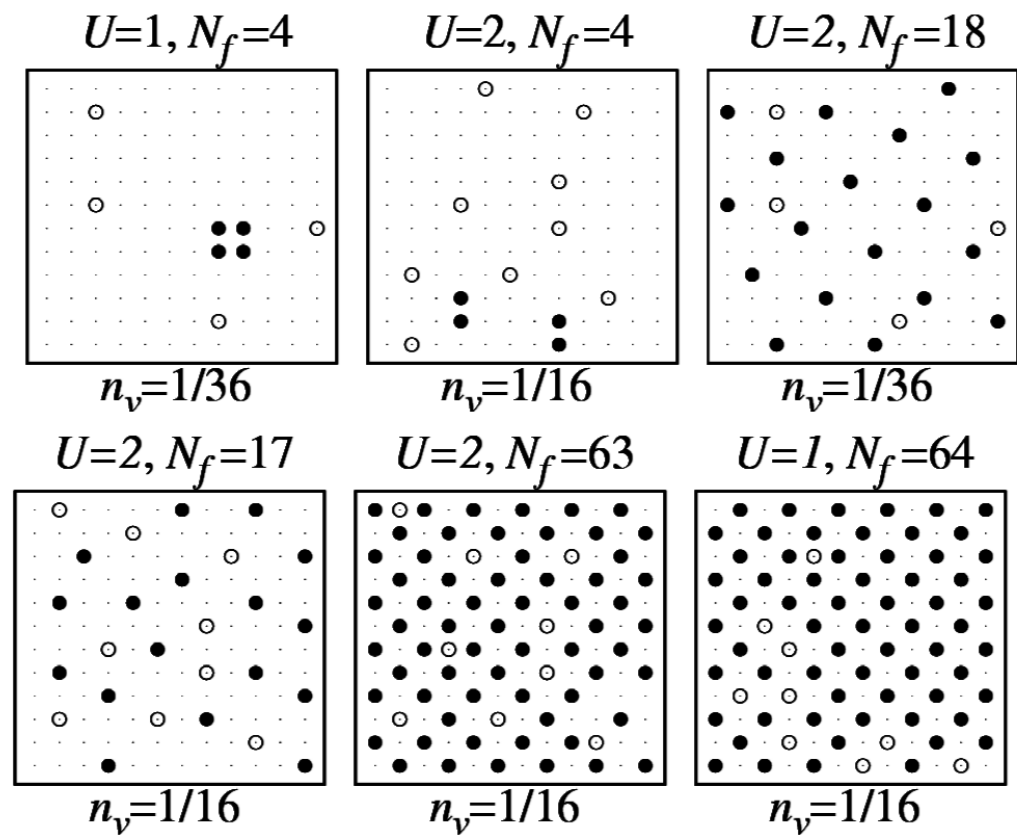

Fig. 1. Representative types of GS configurations for $U=1,2, L=144$ and various $n_{\mathrm{v}}$ and $N_{f}$. $\odot$ represents a vacancy, $\bullet(\cdot)$ represents a position of occupied (unoccupied) $f$ orbital.

the GS configurations are the quasi-homogeneous phases and near $n_{f} \approx 1 / 2$ the perturbed chessboard phases minimise the GS energy. The increase in $U$ and $n_{\mathrm{v}}$ suppresses the area of stability of $n$-molecular phases that vanish entirely for $U>4$ or $n_{\mathrm{v}} \approx 1 / 4$.

Having the complete set of GS configurations for all $f$-electron concentrations $n_{f} \leq 1 / 2$ and all accessible concentrations of vacancies $n_{\mathrm{v}} \leq 1 / 4$ on finite two-dimensional clusters of $L=36$ and $L=64$ sites we have calculated also the average $f$-electron occupancy $n_{f}^{\text {av }}$ as a function of $E_{f}$ by averaging over the set of hundred randomly chosen distributions of vacancies. Typical results of numerical simulations are shown in Fig. 2 and Fig. 3a and they clearly demonstrate that with increasing $n_{\mathrm{v}}$ the staircase structure of the conventional FKM is gradually suppressed and the valence transitions become smoother.

Depending on the values of model parameters $U$ and $E_{f}$ we have observed that $n_{f}^{\text {av }}$ as a function of $n_{\mathrm{v}}$ can increase or decrease. To do the quantitative comparison with experimental data obtained for mixed-valence compound $\mathrm{Sm}_{1-x} \mathrm{~B}_{6}$ [4] we have selected $U=2$ and $E_{f}=0.444$ that models (as our previous results showed [2]) very well the real situation in $\mathrm{SmB}_{6}$ compound $\left(n_{f} \approx 0.47\right)$. The resultant theoretical and experimental behaviours are shown in Fig. 3b and one can see a nice quantitative correspondence between them. This result shows that 


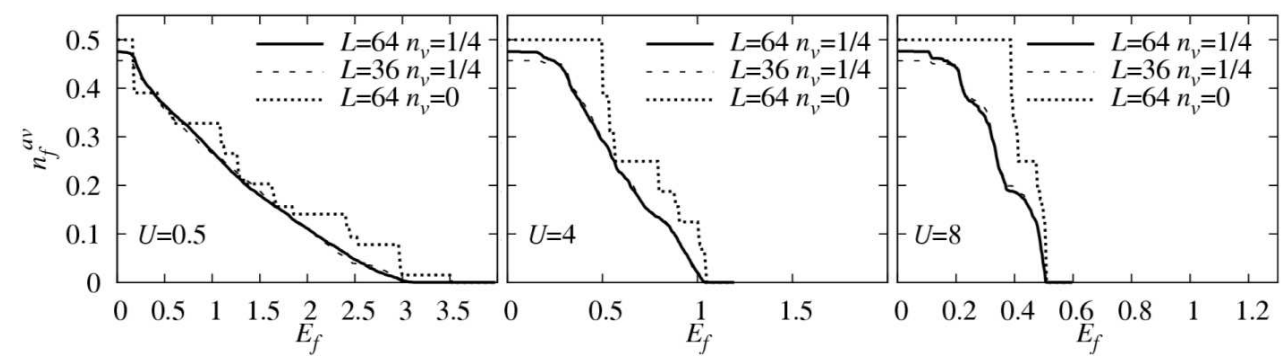

Fig. 2. Dependence of the average $f$-electron occupation number $n_{f}^{\text {av }}$ on the $f$-level position $E_{f}$ for $n_{\mathrm{v}}=1 / 4$ and $n_{\mathrm{v}}=0$ for $U=0.5,4$, and 8 .

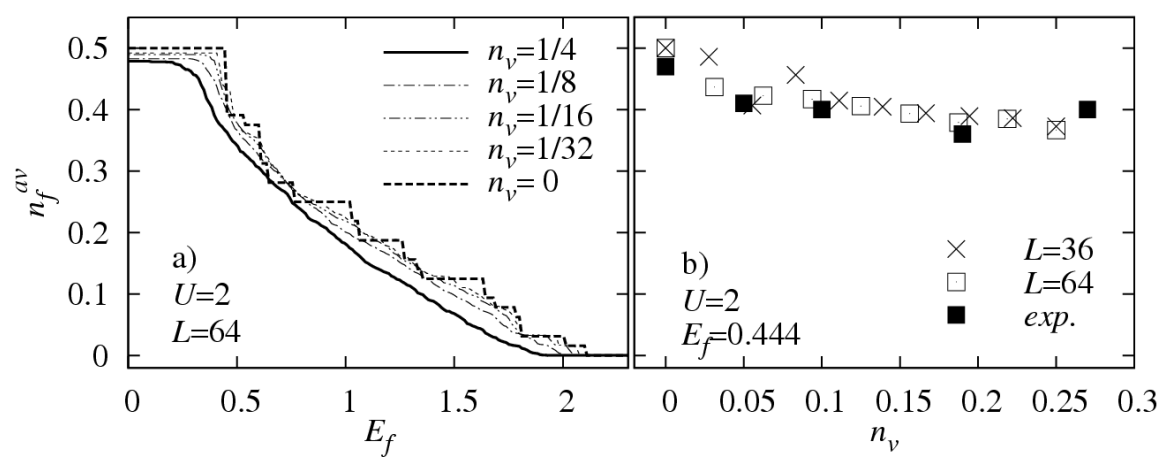

Fig. 3. (a) Dependence of the average $f$-electron occupation number $n_{f}^{\text {av }}$ on the $f$-level position $E_{f}$ for different $n_{\mathrm{v}}$. (b) Dependence of $n_{f}^{\text {av }}$ on $n_{\mathrm{v}}$. (घ) Experimental data obtained for $\mathrm{Sm}_{1-x} \mathrm{~B}_{6}[4],(\times, \bullet)$ theoretical data obtained for $L=36,64$.

the spinless FKM in spite of its relative simplicity can yield the correct physics for description of complex mixed-valence systems, like $\mathrm{Sm}_{1-x} \mathrm{~B}_{6}$.

\section{Acknowledgments}

This work was supported by Slovak Grant Agency VEGA under grant No. 2/7057/27 and Slovak Research and Development Agency (APVV) under grant LPP-0047-06. H.C. acknowledges support of Stefan Schwartz Foundation.

\section{References}

[1] L.M. Falicov, J.C. Kimball, Phys. Rev. Lett. 22, 997 (1969).

[2] P. Farkašovský, Phys. Rev. B 52, 5463 (1995).

[3] R. Lemanśki, J.K. Freericks, G. Banach, Phys. Rev. Lett. 89, 196403 (2002); C.D. Batista, Phys. Rev. Lett. 89, 166403 (2002).

[4] Yu.B. Paderno, E.S. Konovalova, N.L. Baturinskaya, E.M. Dudnik, L.D. Finkelschtein, N.N. Efremova, IAN SSSR Neorg. Mater. 18, 47 (1982).

[5] E.V. Nefedevova, P.A. Alekseev, E.S. Klementev, V.N. Lazukov, I.P. Sadikov, M.N. Khlopkin, M.B. Tsetlin, E.S. Konovalova, Yu.B. Paderno, JETP 88, 565 (1999).

[6] P. Farkašovský, Eur. Phys. J. B 20, 209 (2001). 